\title{
Frequency of Gastrointestinal Diseases in Patients with End-Stage Renal Disease Treated with Long Term Dialysis
}

\section{Lutfi Zylbeari ${ }^{1 *}$, Zamira Bexheti ${ }^{1}$, Gazmend Zylbeari ${ }^{1}$, Ferizat Dika Haxhirexha ${ }^{1}$, Kastriot Haxhirexha ${ }^{1}$}

Received: 08 March 2018; Accepted: 30 June 2018; Published online: 20 July 2018. DOI; https://doi.org/10.32391/ajtes.v2i2.7

\section{Abstract}

Background: Gastrointestinal complications are frequent in patients with renal disease and are responsible for substantial morbidity and mortality among these patients in developing countries. Many times, these patients are subjected to endoscopic evaluation and mucosal biopsies are taken for definitive diagnosis. Long before the routine uses of dialysis, patients dying of uremia were found to have a high incidence of gastrointestinal abnormalities (1).

Matherials and methods: The survey included 240 persons; 120 of them were dialysis patients, while the remaining 120 were healthy individuals who served as a control group. 54 (45\%) of the patients with hemodialysis were females while 66 (55\%) of them were males with mean age: $58.20 \pm 18.00$ years. These patients had been in dialysis for more than 12 years at the Clinic for Nephrology in Skopje and the Clinical Hospital in Tetovo.

Results: Gastrointestinal complications were present in 20 (37.0\%) out of 54 females while 26 (39.4\%) out of 66 males presented with duodenal bulbar ulcers. 84 patients [(females-38/54 (70.4\%) and males-46/66 (85.2\%)] of the total number of 120 examined patients were found to have chronic gastritis.

In conclusion, we found that the incidence of PUD was more than 10 times higher in CKD patients than in those without CKD over a 3-year period between 2008-2010. CKD patients receiving HD, NSAID, or clopidogrel had an increased risk of PUD, compared to CKD patients not receiving these treatments.

Key words: Gastrointestinal disease, chronic gastritis, peptic ulcer disease, hemodialysis.

*Corresponding author: Prof. Lutfi Zylbeari

$\equiv$ E-mail: dr-luti@hotmail.com

1.Faculty of Medical Sciences, University of Tetovo- Republic of Macedonia 


\section{Introduction}

Gastrointestinal (GI) complications are well known in patients with renal disease. The etiologies of $\mathrm{GI}$ complications in CKD are likely to be multifactorial. Urea increases back diffusion of hydrogen ions across the mucosal barrier. Elevated gastrin levels, partly due to the decreased renal clearance of gastrin (4-6) and partly due to the enhanced gastrin synthesis, 6 act as an important stimulus for acid secretion. The above mentioned factors increase gastric mucosal injuries in patients with renal failure. Since gastrin decreases pyloric sphincter tone, high gastrin levels may lead to biliary reflux, thus worsening mucosal injury $(7,8)$. Other mechanisms may contribute to the mucosal abnormalities in ESRD. For example, biliary reflux can worsen gastro-duodenal inflammation. Since gastrin can decrease pyloric sphincter tone, high gastrin levels may worsen biliary reflux, thus worsening mucosal injury. For duodenal ulcers, impaired pancreatic bicarbonate secretion $(35,36)$ and increased pepsinogen release $(9,10)$ in renal failure might also play a role. Several studies have also noted that upper gastrointestinal hemorrhage in dialysis patients is often associated with the use of ulcerogenic drugs such as steroids, iron and especially non-steroidal anti-inflammatory agents, but this is also true in the general population. Although Helicobacter pylori infection is clearly important in causing gastroduodenal lesions in all patients, the infection rates have not been found to be increased in patients with renal failure (11-13,). Gastrointestinal disorders are frequent complications in patients with chronic renal failure treated with hemodialysis.Long before the routine use of dialysis, patients dying of uremia were found to have a high incidence of gastrointestinal abnormalities. Mallory-Weiss tears seem to have a prevalence that is independent of renal function, while esophageal varices may be less frequent in the dialysis population. Patients with renal transplants are at increased risk of esophagitis from opportunistic infections such as Candida, cytomegalovirus and herp/es simplex. Therefore, dialysis patients still tapering immunosuppressive therapy after failed allograft should be carefully watched for these infections. Although uremic patients not on dialysis may have delayed gastric emptying, end stage renal disease itself generally does not cause delayed emptying. However, slower gastric emptying has been documented in patients with intraperitoneal dialysis. Gastrointestinal symptoms related to decreased motility are common in diabetic patients, a group which comprises a large proportion of many dialysis populations.

The routine measurement of gastrin has no clinical role, but the literature documenting gastrin elevations in renal failure should be kept in mind if searching for a gastrinoma in a dialysis patient (1418).Improvements in dialysis technique and changes in the patient population undergoing dialysis have modified the spectrum of gastrointestinal diseases seen in patients with endstage renal disease (ESRD), also called chronic kidney failure, a term that describes the gradual loss of kidney function.

\section{Matherials and methods}

The survey included 240 individuals in total. Half of them (120 patients) were dialysis patients and the other half were healthy individuals who served as a control group. Overall our "cross-sectional" study included, 108 (45\%) women of average age of 59.50 \pm 14.50 years and $132(55 \%)$ men of average age of $=56.40 \pm 12.80$ years. The gender distribution for the dialysis patients was as follows: 54 (45\%) of them 
were females and the remaining 66 (55\%) were males, with mean age: $58.20 \pm 18.00$ years. The patients had been on dialysis for more than 12 years in the Nephrology Clinic -Skopje and the Tetovo Clinical Hospital. The control group of healthy examiners (volunteer blood donors) had also the same female to male ratio: 54 (45\%) females : 66 (55\%) males. The two groups were also the same concerning their age, gender, religious affiliation and national affiliation. All patients with gastrointestinal complications examined $\mathrm{H}$ : $\mathrm{B}$, as well as a small number of gastroscopies.

\begin{tabular}{r|ll} 
Gender & Number & \multicolumn{1}{l}{$\begin{array}{l}\text { The Average } \\
\text { age } \pm S D\end{array}$} \\
\hline Men & $66(55 \%)$ & $56.40 \pm 12.80$ \\
\hline Females $54(45 \%)$ & $59.50 \pm 14.50$
\end{tabular}

Table no 1. Number of patients presented by sex and average age

\begin{tabular}{llll|l}
\multirow{2}{*}{ Gender } & \multicolumn{2}{l}{$\begin{array}{l}\text { Macedonian } \\
\text { (45\%) }\end{array}$} & \multicolumn{3}{c}{$\begin{array}{l}\text { Albanian } \\
\text { (55\%) }\end{array}$} \\
\cline { 2 - 5 } Men & $\mathrm{Nr}$ & $\%$ & $\mathrm{Nr}$ & $\%$ \\
Females & 30 & 25.0 & 36 & 30.0 \\
\cline { 2 - 5 } & 24 & 20.0 & 30 & 25.0
\end{tabular}

Table no 2: Distribution of patients according to nationality

Out of the total number of patients, $54(45 \%)$ of them were Macedonians and 66 (55\%) were Albanians. The gender distribution for the Macedonian patients (45\%) was as follows: $25 \%$ males and $20 \%$ females. The male to female ratio for Albanian patients was $30 \%$ males: $25 \%$ female.
Concerning their religious affiliation, 66 (55\%) patients were of Islamic faith and 54 (45\%) of the Catholic religion.

\section{Statistical analysis of the examined material}

Statistical basic methods such as the arithmetic mean value and standard deviation ( $\mathrm{X} \pm \mathrm{SD}$ ) were used. Comparative statistics parameters between the two groups were analyzed by using specific statistical tests called StudentoV, Mann-Whitney and Wilcoxon's test. The results were presented in the form of tables and in the form of processed diagrams.

\section{Results}

Gastrointestinal disorders are frequent complications in patients with chronic renal failure undergoing hemodialysis. In our study gastrointestinal complications were present in 20 (37.0\%) out of 54 females while 26 (39.4\%) out of 66 males presented with duodenal bulbar ulcers. 84 patients [(females-38/54 (70.4\%) and males-46/66 (85.2\%)] of the total number of 120 examined patients were found to have chronic gastritis, verified by clinical, ventricular, gastroscopic and CLO-tests. In the group of examiners a positive presence of serum $\mathrm{HbsAg}$ was observed in 10 (14.8\%) females and 12 (18.2\%) males. Anti HbsAgpositivity was found in 26/54 (48.1\%) of females and $35 / 66(57.6 \%)$ of males. Those findings were result of contact and previous infection with Hepatitis-B. Virus virion of the manifested clinical hepatitis was not observed in the group of examined patients. 


\begin{tabular}{r|lll|l} 
Gastrointestin & \multicolumn{2}{l}{ Females } & \multicolumn{2}{l}{ Men } \\
\cline { 3 - 5 } al & Nr. & $\%$ & Nr. & $\%$ \\
complications & & & & \\
\hline $\begin{array}{r}\text { Duodenal } \\
\text { ulcer }\end{array}$ & 20 & 37,0 & 26 & 39.4 \\
$\begin{array}{r}\text { Chronic } \\
\text { gastritis }\end{array}$ & 38 & 70.4 & 46 & 85.2 \\
Ascites & 8 & 14.8 & 12 & 18.2
\end{tabular}

Table no. 3. Gastrointestinal complications of patients treated with hemodialysis

The highest percentage of gastrointestinal complications of the examined patients were chronic gastritis with $=82.8 \%$, duodenal ulcers with $=79.4 \%$ and ascites with $=38.2 \%$ (tabl. 3 ).

\section{Discussion}

CKD patients receiving hemodialysis treatment comprise more than 1.2 million people in the world, and this population is expanding at a rate of $7 \%$ per year according to the progress in medical and dialysis technology.1 GI complications are known to commonly occur in patients with renal failure. Uremia and dialysis have been found to increase the risk of lesions in the GI tract. Gastrointestinal disorders are frequent complications in patients with chronic renal failure treated with hemodialysis. Long before the routine use of dialysis, patients dying of uremia were found to have a high incidence of gastrointestinal abnormalities.

Mallory-Weiss tears seem to have a prevalence that is independent of renal function, while esophageal varices may be less frequent in the dialysis population. Patients with renal transplants are at increased risk of esophagitis from opportunistic infections such as Candida, cytomega-lovirus and herples simplex. Therefore, dialysis patients still tapering immunosuppressive therapy after failed allograft should be carefully watched for these infections. While uremic patients not on dialysis may have delayed gastric emptying, end stage renal disease itself generally does not cause delayed emptying. However, slower gastric emptying has been documented with inperitoneal dialysis patients during a dialysis fluid dwell period. Furthermore, gastrointestinal symptoms related to decreased motility are common in diabetic patients, a group which comprises a large proportion of many dialysis populations. Most investigators have concluded that gastrin elevation does not explain the mucosal lesions in patients with ESRD. The routine measurement of gastrin has no clinical role, but the literature documenting gastrin elevations in renal failure should be kept in mind for the possibility of gastrinoma in a dialysis patient (1418).

Improvements in dialysis technique and changes in the patient population under-going dialysis have modified the spectrum of gastrointestinal diseases seen in patients with end-stage renal disease (ESRD), also called chronic kidney failure, a term that describes the gradual loss of kidney function. Peptic ulcer disease (PUD) is still a common disease in elderly patients and patients with multiple comorbid conditions. Evidence suggests that Helicobacter pyloriinfection and use of nonsteroidal anti-inflammatory drugs (NSAID) are the primary causes of PUD in the general population. However, compared to the general population, patients with chronic kidney disease (CKD) have distinct causative factors and clinical outcomes of gastro-duodenal ulcers. Population-based studies have demonstrated that CKD patients have a higher risk of peptic ulcer bleeding and bleeding-related 
morbidity and mortality. Tseng et al. reported a high recurrence rate of PUD among hemodialysis (HD) patients even after $H$. pylori eradication. Another longitudinal study also reported that PUD occurred in a significant number of long-term HD patients despite a low prevalence of $H$. pylori nfection. Both PUD and CKD are leading publichealth issues, and many studies have described associations between them (19-29). Despite this, limited information is available about temporal trends in PUD among CKD patients $(5,6,7)$. Another limitation of existing evidence is the focus on hospitalized patients with peptic ulcer bleeding, preventing generalization to the entire CKD population . It is also unclear if CKD patients differ from non-CKD peptic ulcer patients with respect to the ulcer location (gastric or duodenal mucosa) and patient status (inpatient or outpatient). Finally, it remains uncertain whether CKD patients taking ulcerogenic medications (e.g., NSAIDs or aspirin) are more likely to develop PUD $(30,31)$. In addition, we determined the incidence of PUD over a 10-year period, compared between CKD patients and patients without CKD. More specifically, the effects of gastroduodenal mucosa and ulcerogenic medications on CKD-related PUD were investigated. Dialysis treatment, including the mode and length of the treatment, was recorded.

Because the different dialysis modalities can affect the risk of ulcerous duodenal CKD patients were specifically divided into patients who had never received HD treatment (non-HD CKD) and patients who received $H D$ treatment at any time, regardless of the duration of treatment (HD-CKD). HD-CKD patients included patients who had HD for $<3$ months (temporarily HD-CKD) and those who underwent $\mathrm{HD}$ for $\geq 3$ months (HD-CKD maintenance). Depending on the location of the ulcer and the upper part of the endoscopy (eg, in the outpatient department or during hospitalization), these groups were further stratified to observe the effect of CKD on the stomach and duodenal mucosa. H. pylori peptic ulcer has been identified by receiving $\mathrm{H}$. pylori eradication therapy during or after the date of the index. H. pylori eradication was defined as administration of proton pump inhibitors or $\mathrm{H} 2$ receptor antagonists plus clarithromycin or metronidazole plus amoxicillin or tetracycline and with or without bismuth. The use of drugs has been documented for aspirin, NSAIDs (except aspirin), warfarin, clopidogrel, and cilostazol. NSAIDs consisted of cyclooxygenase-2-specific inhibitors (COXIBs) and traditional NSAIDs (except COXIBs (24-28)). More importantly, there was a rapid increase in the incidence of PUD in elderly patients with CKD, compared to a decrease in younger CKD patients. Several factors, including HD therapy, patient access (inpatient vs. outpatient), and the use of NSAID and clopidogrel, further affected peptic ulcer risk in CKD patients. In addition, CKD patients undergoing mainte-nance HD were likely to develop GU following long-term $H D$ therapy. Overall, we suggest that CKD itself is a strong independent risk factor for PUD, and the incidence of PUD among elderly CKD patients is substantially increasing.Well-developed studies have demonstrated that CKD patients with peptic ulcer bleeding were more likely to have adverse outcomes such as prolonged hospital days and increased mortality rates $(111,18,19)$.The incidence of PUD in the general population is reported to be decreasing, while our results indicate that the incidence is increasing in patients with CKD. The decrease in PUDs in the general population may be the result of a decrease in $H$. pylori infections (18). Given that the prevalence of $H$. pylori infection in CKD patients is lower than in those with normal renal function (7), the increase in PUD in this population is likely due to other causes. In recent 
decades, CKD has been associated with increasing age, the presence of a greater number of comorbidities, and an increased use of $\operatorname{NSAID}(21,22)$. Therefore, we thought that these factors are probable explanations for this increasing incidence of PUD in CKD, particularly as they are also well-known risk factors for PUD.Our results indicate a deleterious influence of HD therapy on peptic ulcer risk, regardless of dialysis duration. Anticoagulant use during HD may contribute to this risk $(3,6,9,10)$. In addition, intradialytic hypotension and hemodynamic changes might play a role in the occurrence of PUD. Intradialytic hypo-thension remains one of the most common $\mathrm{HD}$ problems and occurs in approximately $20-30 \%$ of HD sessions (23).The majority of CKD cases (96.3\%) in this study received endoscopic diagnoses and treatments in the inpatient setting instead of the outpatient department. Yang et al indicated that approximately $10 \%$ of upper gastrointestinal bleeding episodes in the dialysis population were managed in the ambulatory care department (5). It should be noted that PUD may be the primary cause of admission or may be a complication in those already hospitalized for another condition . Even though CKD patients are considered to have a high risk for ulcer rebleeding and require more surveillance in hospitals $(4,12)$, this heigh-tened awareness does not fully explain this result.Further studies might be warranted to investigate the effects of the above factors on the association between CKD and PUD. CKD patients receiving hemodialysis trea-tment consist of more than 1.1 million people in the world, and the size of this population is expanding at a rate of $7 \%$ per yearaccording to the progress in medical and dialysis machine technique. GI complications are known to commonly occur in patients with renal failure. Uremia and dialysis have been found to increase the risk of lesions in the Gl tract.
Predominant biopsy findings included erosive gastritis, ulcerative esophagitis, esophageal candidiasis, and duodenitis/duodenal ulcerations. None of these patients were documented to be on any gastric irritant drugs.

The low incidence of esophageal candidiasis in this study is probably due to the fact that they are not routinely biopsied in our hospital. This study exposes the wide spectrum of GI pathology in patients with kidney disease. Opportunistic infections are well known in allograft recipients; however, morphological effects of drug toxicity remain underreco-gnized since these patients are not always biopsied. With the emerging reports of rather specific drug-related biopsi changes, endoscopic mucosal biopsies play unquestionable role in the management of these patients. H. pylori is a major cause of gastritis in the gjeneral population. $\mathrm{H}$. pylori is a major cause of gastritis in the gjeneral population. The increase in the availability of urea, asubstrate for $\mathrm{H}$. pylori metabolism, might be expected to increase the prevalence of $\mathrm{H}$. pylori infection in the CKD population. The prevalence of infection decreases as dialysis periods progressed, in particularwithin the first 4 years after the start of treatment, suggestingthat hemo-dialysis treatment, but not uremia, plays a role in the lower prevalence of $\mathrm{H}$. pylori infection(32-37). Renal failure patients on hemodialysis or peritoneal dialysis are prone to develop this complication due to repeated bouts of hypotension during dialysis.

\section{Conclusions}

In conclusion, we found that the incidence of PUD was more than 10 times higher in CKD patients than in those without CKD over a 3-year period between 2008-2010. CKD patients receiving HD, NSAID, or 
clopidogrel had an increased risk of PUD, compared to CKD patients not receiving these treatments. Peptic ulcer risk might be influenced by ulcer location, HD therapy, inpatient status, and ulcerogenic medications.

\section{References}

1. Zuckerman GR, Cornette GL, et al. Upper gastrointestinal bleeding in patients with chronic renal failure. Ann Intern Med 102: 588, 1985.

2. Margolis DM, Saylor JL, et al. Upper gastrointestinal disease in chronic renal failure: a prospective evaluation. Arch Intern Med 138: 1214, 1978.

3. McNamee PT, Moore GW, McGeown M, Doherty CC, Collins BJ: Gastric emptying in chronic renal failure. Br Med J 291:310, 1985.

4. Gold CH, Morley JE, Viljoen M, et al. Gastric acid secretion and serum gastrin levels in patients with chronic renal failure on regular hemodialysis. Nephron. 1980;25:92.

5. Muto S, Murayama N, Asano Y, et al. Hypergastrinemia and achlorhydria in chronic renal failure. Nephron. 1985;40:143.

6. Ghonaimy E, Barsoum R, Soliman M, et al. Serum gastrin in chronic renal failure: morphological and physiological correlation. Nephron. 1985;39:86.

7. Paronen I, Ala-Kaila K, Rantala I, et al. Gastric parietal, chief, and G-cell densities in chronic renal failure. Scand J Gastroenterol. 1991;26:696-700. 8. Fisher RS, Lipshutz W, Cohen S. The hormonal regulation of pyloric sphincter function. J Clin Invest. 1973;52:1289.

9. McNamee PT, Moore GW, et al. Gastric emptying in chronic renal failure. Br Med J 291:310, 1985 10. Wright RA, Clemente R, Wathen R: Gastric emptying in patients with chronic renal failure receiving hemodialysis. Arch Intern Med 144: 495, 1984
11. Soffer EE, Geva B, et al. Gastric emptying in chronic renal failure patients on hemodialysis. J Clin Gastroenterol 9: 651, 1987

12. Brown-Cartwright $D$, Smith HJ, Feldman M: Gastric emptying of an indigestible solid in patients with endstage renal disease on continuous ambulatory peritoneal dialysis. Gastroenterology 95: 49, 1988 13. Feldman M, Schiller LR: Disorders of gastrointestinal motility associated with diabetes mellitus. Ann Intern Med 98: 378, 1983.

14. Franzin G, Musola R, Mencarelli R: Morphological changes of the gastroduodenal mucosa in regular dialysis uraemic patients. Histopathology 6: 492, 1982. 15. Zuckerman GR, et al. Upper gastrointestinal bleeding in patients with chronic renal failure. Ann Intern Med 102: 588, 1985

16. Malfertheiner $P$, Chan FK, et al. Peptic ulcer disease. Lancet,2009; 374: 1449-14526.

17. Luo JC, Leu HB, Huang KW, et al. Incidence of bleeding from gastroduodenal ulcers in patients with end-stage renal disease receiving hemodialysis.2012; CMAJ 183: E1345-1352. 18. Tseng GY, Lin HJ, Fang CT, et al. Recurrence of peptic ulcer in uraemic and non-uraemic patients after Helicobacter pylori eradication: a 2-year study.2007; Aliment Pharmacol Ther 26: 925-933. 19. Tseng GY, Lin HJ, Fang CT, et al. Recurrence of peptic ulcer in uraemic and non-uraemic patients after Helicobacter pylori eradication: a 2-year study.2007; Aliment Pharmacol Ther 26: 925-933. 20. Sugimoto $M$, Sakai $K$, et al. Prevalence of Helicobacter pylori infection in long-term hemodialysis patients. (2009) Kidney Int 75: 96104.

21. Wen CP, Cheng TY, et al. All-cause mortality attributable to chronic kidney disease: a prospective cohort study based on 462293 adults in Taiwan.2008; Lancet 371: 2173-2182. 
22. Barkun A, Leontiadis G. Systematic review of the symptom burden, quality of life impairment and costs associated with peptic ulcer disease. (2010) Am J Med 123: : 358-366

23. Chen YT, Yang WC, et al. Comparison of peptic ulcer disease risk between peritoneal and hemodialysis patients. 2010. Am J Nephrol 32: 212218

24. Wasse H, Gillen DL, Ball AM, et a. Risk factors for upper gastrointestinal bleeding among endstage renal disease patients. (2003) Kidney Int 64: 1455-1461

25. Hsu PI, Lai KH, Liu CP Esomeprazole with clopidogrel reduces peptic ulcer recurrence, compared with clopidogrel alone, in patients with atherosclerosis. (2011) Gastro-enterology 140: 791-798]

26. Hsu PI New look at antiplatelet agent-related peptic ulcer: An update of prevention and treatment. (2012) Journal of Gastroenterology and Hepatology 27: 654-661

28. Peng YL, Leu HB, Luo JC, et al.Diabetes is an independent risk factor for peptic ulcer bleeding: a nationwide population-based cohort study. (2013) Journal of Gastroenterology and Hepatology,648730.

29. Ethier J, Bragg-Gresham JL, Piera L, Akizawa T, Asano $Y$, et al. Aspirin prescription and outcomes in hemodialysis patients: the Dialysis Outcomes and Practice Patterns Study (DOPPS). (2007), Am J Kidney Dis 50: 602-615 30. Laine L, Maller ES, Yu C, Quan H, Simon T. Ulcer formation with low-dose enteric-coated aspirin and the effect of COX-2 selective inhibition: a doubleblind trial. (2004) Gastroenterology 127: 395-406 31. Éthier J, Bragg-Gresham JL, Piera L, Akizawa T, Asano $Y$, et al. (2007) Aspirin prescription and outcomes in hemodialysis patients: the Dialysis Outcomes and Practice Patterns Study
(DOPPS). American Journal of Kidney Diseases 50: 602-615.

32. Chong VH. Impact of duration of hemodialysis on gastrointestinal symptoms in patients with end stage renal failure. J Gastrointestin Liver Dis. 2010;19(4):462-463.

33. Flobert $C$, Cellier C, Berger A, et al. Right colonic involvement is associated with severe forms of ischemic colitis and occurs frequently in patients with chronic renal failure requiring hemodialysis. Am J Gastroenterol. 2000;95(1): 195-198 34. Dalle IJ, Maes BD, Geboes KP, et al. Crohn's-like changes in the colon due to mycophenolate? Colorectal Dis. 2005;7:27-34

35. Lysaght MJ. Maintenance dialysis population dynamics: current trends and long-term implications. J Am Soc Nephrol. 2002;13 (Suppl. I):S37-S40. [2] Khedmat H, Ahmadzad-Asl M, 36. Amini $\mathrm{M}$, et al. Gastroduodenal lesions and Helicobacter pylori infection in uremic patients and renal transplant recipients. Transplant Proc. 2007;39(4):1003-1007. [3] Gold CH, Morley JE, Viljoen $\mathrm{M}$, et al. Gastric acid secretion and serum gastrin levels in patients with chronic renal failure on regular hemodialysis. Nephron. 1980;25:92. [4] ] 37. Paronen I, Ala-Kaila K, Rantala I, et al. Gastric parietal, chief, and G-cell densities in chronic renal failure. Scand J Gastroenterol. 1991;26:696-700. 